\title{
Blunt cerebrovascular injury: diagnosis at whole-body MDCT for multi-trauma
}

\author{
Matteo Bonatti • Norberto Vezzali • Federica Ferro • \\ Riccardo Manfredi • Nadia Oberhofer • \\ Giampietro Bonatti
}

Received: 29 December 2012 /Revised: 10 February 2013 / Accepted: 12 February 2013 /Published online: 21 March 2013

(C) The Author(s) 2013. This article is published with open access at Springerlink.com

\begin{abstract}
Purpose To analyse the prevalence of blunt cerebrovascular injuries (BCVIs) in multi-trauma patients by means of a post-contrast acquisition of neck vessels included into the whole-body multi-detector computed tomography (MDCT) protocol performed at admission and to correlate it with the presence of risk factors (Memphis approach).

Materials and methods A retrospective study was undertaken for the period January 2005 to November 2011, involving 976 multi-trauma patients. Post-contrast images of neck vessels in MDCT scan were evaluated by two experienced radiologists; carotid, vertebral and basilar arteries were rated according to the Biffl classification. The presence of clinical and/or CT risk factors for BCVI was assessed.

Results BCVI were present in 32/976 (3.3 \%) multi-trauma patients. Risk factors for BCVI were present in 247/976 $(25.3 \%)$ patients. The group of patients presenting risk factors showed a significantly higher prevalence of cerebrovascular injuries $(8.1 \%)$ compared with the group of patients without risk factors $(1.6 \%)(p=0.009)$; however, $12 / 32(37.5 \%)$ patients presenting BCVI did not show any of the risk factors proposed by the Memphis group.
\end{abstract}

M. Bonatti $(\bowtie) \cdot$ N. Vezzali $\cdot$ F. Ferro $\cdot$ G. Bonatti

Department of Radiology, San Maurizio Hospital,

5 Boehler Street, 39100 Bolzano, Italy

e-mail: matteobonatti@hotmail.com

R. Manfredi

Department of Radiology, Policlinico G.B. Rossi,

10 L.A. Scuro Place, Verona, Italy

N. Oberhofer

Department of Physics, San Maurizio Hospital,

5 Boehler Street, Bolzano, Italy
Conclusion An investigation for the presence of BCVI should be performed on all multi-trauma patients despite the absence of clinical-radiological risk factors.

Key Points

- BCVIs are present in $3.3 \%$ of multi-trauma patients.

- BCVIs are significantly associated to the Memphis risk factors.

- Of the multi-trauma patients affected by BCVIs, $37.5 \%$ do not show clinical-radiological risk factors.

- A screening for BCVI should be performed on all multitrauma patients.

Keywords Carotid artery injury · Vertebral artery dissection - Multiple trauma - Spiral computed tomography · Spine

\section{Introduction}

Blunt cerebrovascular injuries (BCVIs) are considered relatively uncommon in patients admitted to an emergency department because of blunt trauma, with reported incidences of $0.5-1.5 \%$, but their incidence increases to about $2.7 \%$ if only patients with an Injury Severity Score (that is the most widely adopted anatomical scoring system for grading the severity of trauma in patients with multiple injuries) greater than 16 are considered [1-7].

Clinical presentation of BCVI is similar to spontaneous dissections $[8,9]$. Many BCVIs are asymptomatic in their early phases and they can be easily missed at initial evaluation, particularly in severely traumatised patients [1, 10-14].

The outcomes of BCVI can be devastating, including debilitating stroke and death, with mortality rates of 8 $38 \%[3,7,9,15,16]$. Although some conflicting results 
exist [17], it is quite well established that an early treatment improves the outcome of patients affected by BCVI, reducing stroke and mortality rates [3, 7, 9, 18-25]. Therefore, an early diagnosis is fundamental.

Because of its high sensitivity and specificity, four-vessel cervicocerebral digital subtraction angiography (DSA) is considered the "gold standard" for the diagnosis of BCVI [26]; however, thanks to the recent technical developments, multi-detector computed tomography (MDCT) angiography is nowadays regarded as an acceptable screening method for BCVI [27-31]. Moreover, the recent works by Sliker [30] and Rademacher [32] demonstrated that a post-contrast acquisition of the neck included into a whole-body MDCT protocol can be considered an acceptable initial means of screening multi-trauma patients for the presence of BCVI.

Many different algorithms have been developed In order to select which blunt-injured patients need to be aggressively investigated for the presence of BCVI; the ones proposed by the groups of Denver [33] and Memphis [20] being the most widely accepted. All these algorithms are substantially based on the presence of clinical/radiological risk factors for BCVI [13], but they seem to lack in sensitivity, because it has been proven that BCVI can occur even in the absence of clinical-radiological risk factors [4, 6, 7, 34, 35].

The aim of our study was to analyse the prevalence of BCVIs in a severely injured patient population, represented by patients classified as multi-trauma, by means of a postcontrast acquisition of neck vessels included in the wholebody MDCT examination that these patients routinely undergo at admission to our institution, and to correlate the presence of BCVI to the presence of the risk factors proposed by the Memphis group.

\section{Materials and methods}

\section{Patient population}

Our retrospective study was approved by the Investigational Review Board of our Institution; informed consent was waived.

In the period January 2005 to November 2011, 1153 patients admitted to the Emergency Department of our Institution have been classified as multi-trauma, according to their clinical situation (two or more injuries, of which at least one life-threatening or Injury Severity Score $\geq 16$ ) and/or to the trauma dynamic (high-speed trauma or fall from an height $>3 \mathrm{~m}$ ), and therefore they have been considered eligible for the performance of a whole-body MDCT according to our "multitrauma protocol". We considered for inclusion into our study the 994/1153 (86.2\%) patients who underwent an MDCT examination within the first $12 \mathrm{~h}$ from admission; whereas we excluded the 52/1153 (4.5\%) patients who underwent the MDCT $>12 \mathrm{~h}$ from admission and the $107 / 1153(9.3 \%)$ patients who did not undergo MDCT because of death. Eighteen out of $994(1.8 \%)$ patients were excluded because of insufficient diagnostic quality of the post-contrast cervical vessels acquisition due to motion artefacts or technical problems. Thus, our study population encompassed 976 patients, 770 men $(78.9 \%)$ and 206 women $(21.1 \%)$, with a median age of 43 years (range 18-96 years).

Mechanisms of injury were car (331/976, $33.9 \%)$ or motorcycle crash (233/976, $23.9 \%)$, skiing (201/976, $20.6 \%$ ) or bicycle accident (64/976, $6.6 \%$ ), pedestrian struck $(50 / 976,5.1 \%)$, fall from a great height $(42 / 976$, $4.3 \%$ ) and other less common mechanisms (55/976, $5.6 \%)$.

Clinical data at the time of admission were retrieved from our institutional informatics system.

Imaging technique: whole-body MDCT multi-trauma protocol

All the CT examinations were performed on a 16-row MDCT scanner (Somatom Sensation 16, Siemens Medical Systems, Erlangen, Germany). The patient was placed supine on the table, with the arms lying down along the body as close as possible to the abdomen in order to reduce beam hardening artefacts.

The first step of our standard whole body MDCT multitrauma protocol is a plain helical acquisition of the head, including the mandible, with the following scanning parameters: $16 \times 0.75 \mathrm{~mm}$ collimation, $120 \mathrm{kV}$ tube voltage, $320 \mathrm{mAs}$ tube current and pitch of 0.55 .

After that we administer, through a peripheral venous access of at least $18 \mathrm{G}, 120-150 \mathrm{ml}$ iodine-containing contrast medium (Iomeprolo, Iomeron350; Bracco, Milano, Italy) using a power injector (Spectris; Medrad, Pittsburgh, Pennsylvania, USA) at a flow rate of $4.0 \mathrm{ml} / \mathrm{s}$, followed by a saline flush of $50 \mathrm{ml}$ at a flow rate of $4.0 \mathrm{ml} / \mathrm{s}$.

The post-contrast scan is acquired using a bolus tracker placed in the aortic arch, with a threshold trigger of $100 \mathrm{HU}$ and a delay trigger of $9 \mathrm{~s}$ and extends cranio-caudally from the skull basis to the femoral lesser trochanters, with a $16 \times 1.5-\mathrm{mm}$ collimation and a pitch of 1.15 , using the CARE dose 4D automatic exposure control (Siemens Medical Systems). Moreover a portal venous phase scan of the abdomen is acquired with a fixed delay of $70 \mathrm{~s}$ after injection start, extending craniocaudally with a $16 \times 1.5-\mathrm{mm}$ collimation, pitch 0.75 and using CARE dose 4D automatic exposure control modality.

According to the clinical question and to the imaging findings, additional pre- and/or post-contrast acquisitions can be performed.

The obtained axial source images are reformatted as multi-planar reconstructions (MPRs) and maximum intensity projections (MIP) with different kernels and slice thicknesses according to the different anatomical districts; in particular, the axial source images of craniocervical vessels 
are reformatted as 2-mm MPRs with a reconstruction increment of $1 \mathrm{~mm}$, using a sharp kernel.

\section{Image analysis}

Two radiologists (with 22 and 12 years of experience in emergency radiology, respectively), who were unaware of the trauma dynamics and of the clinical conditions of the patient, independently evaluated the post-contrast CT images of cerebrovascular vessels. Discrepancies were solved by consensus. Common carotid arteries, internal carotid arteries, vertebral arteries and basilar artery were evaluated on 2-mm MPR images and each vessel was classified as follows, according to the angiographic classification proposed by Biffl et al. [36] (Figs. 1, 2, 3, 4 and 5): normal (grade 0), lumen wall irregularity or lumen narrowing $<25 \%$ (grade I) (Fig. 1a); intraluminal thrombus or lumen
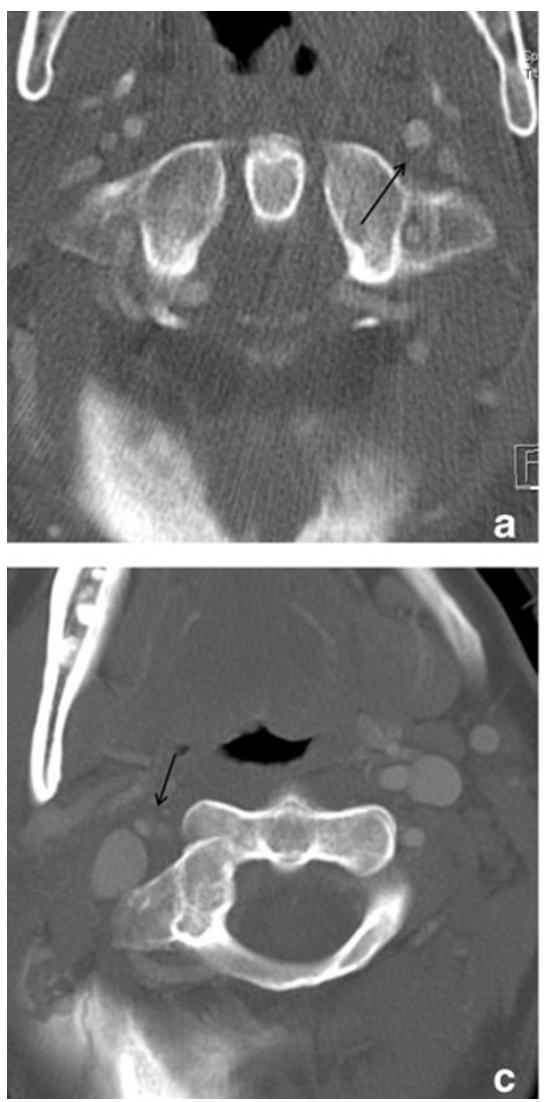

Fig. 1 Low-grade blunt cerebrovascular injury (BCVI) (grade I and II lesions according to the Biffl classification). a A 42-year-old man who had suffered a motorcycle accident. The axial MPR image (3-mm thickness) shows a slight irregularity in the posterior wall of the left internal carotid artery (arrow) responsible of a calibre reduction $<25 \%$ (grade I lesion). No clinical-radiological risk factors for BCVI were present. The man did not undergo anti-aggregation because of the presence of severe abdominal injuries and developed an infarction in the territory of the left median cerebral artery. b An 83-year-old woman struck by a car. The axial MPR image (3-mm thickness) shows an eccentric calibre reduction of the left vertebral artery (arrow) $>25 \%$ narrowing $>25 \%$ (grade II) (Fig. 1b-d); pseudoaneurysm (grade III) (Fig. 2a-d); complete occlusion (grade IV) (Fig. 3a-d); transection associated to active extravasation of contrast media (grade V) (Fig. 4a-c).

A third radiologist (with 3 years of experience in emergency radiology), aware of the trauma dynamics and of the clinical conditions of the patient, searched for the presence of risk factors for BCVI: cervical spine fractures, Le Fort II or III facial fractures, skull base fractures involving the foramen lacerum and CT signs of diffuse axonal injury (considered as the presence of five or more small petechial haemorrhages located at grey-white matter junction and in corpus callosum).

\section{Statistical analysis}

The inter-observer variability was assessed by means of kappa statistic for the qualitative image analysis parameters
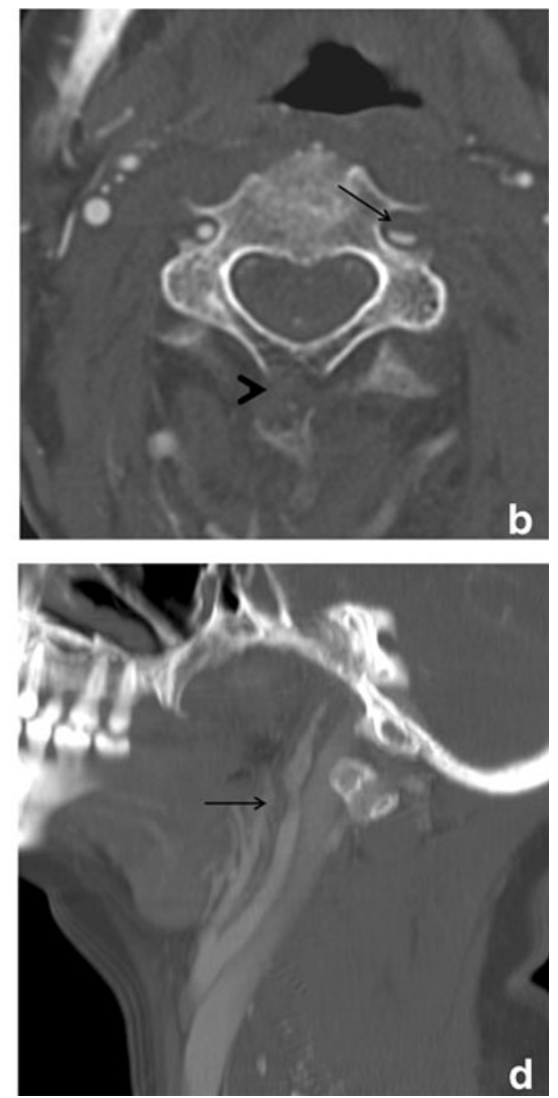

(grade 2 lesion). Cervical spine fractures, including a fracture of the spinous process of C3 (arrowhead) were present. The vertebral artery lesion had not been described on the radiological report and, therefore, no therapy had been administered. The woman developed a cerebellar infarction. c, d A 34-year-old man who had suffered a motorcycle accident. The axial MIP reconstruction (c) (3-mm thickness) shows a lumen wall irregularity of the right internal carotid artery (arrow) responsible of a calibre reduction $>25 \%$ (grade IIlesion); the lesion is better depicted on the sagittal MIP reconstruction $\mathbf{d}$ (5-mm thickness). No clinical-radiological risk factors for BCVI were present. The men underwent anti-aggregation and no neurological deficit occurred 

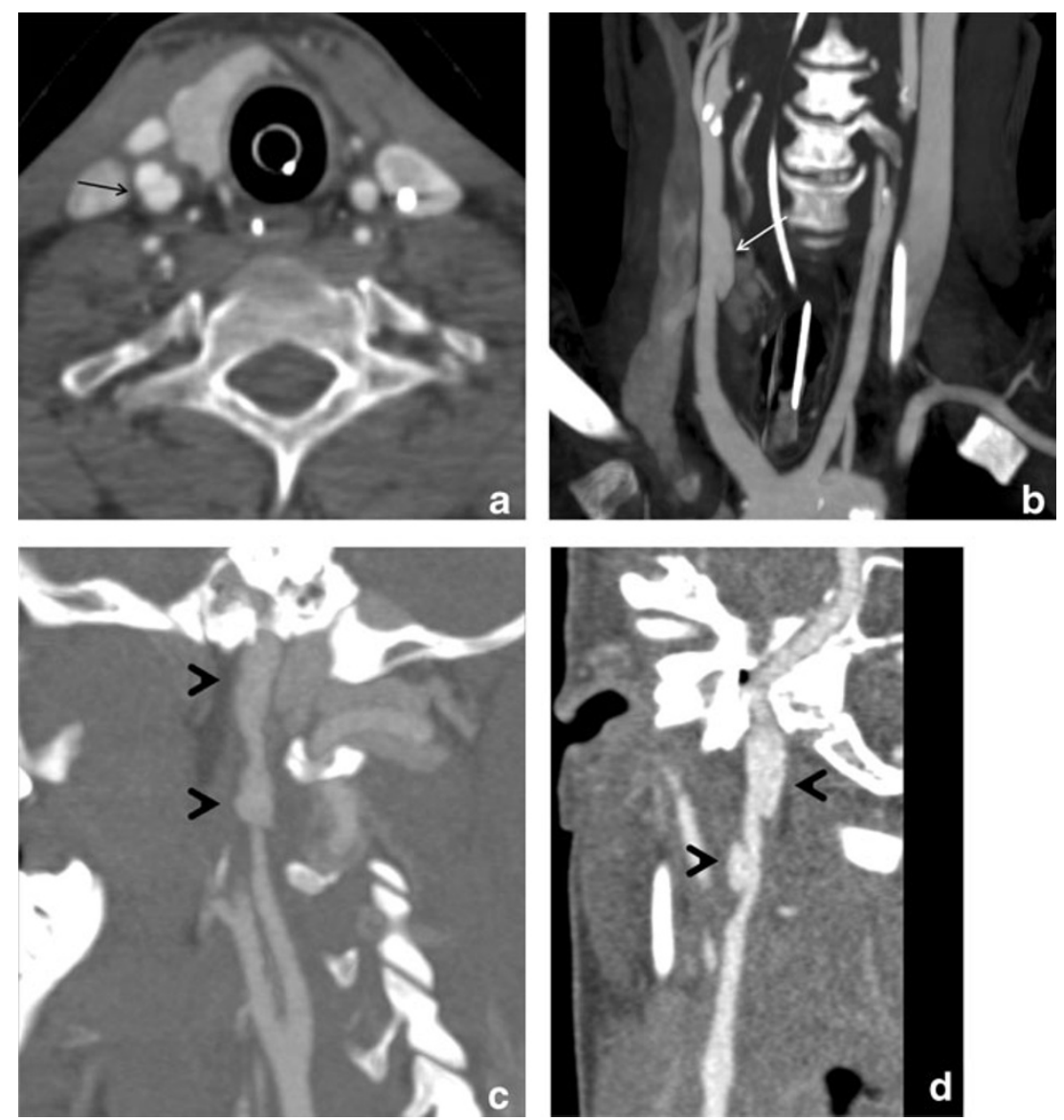

Fig. 2 Blunt cerebrovascular pseudoaneurysm (grade III lesions according to the Biffl classification). a, b A 57-year-old man who had suffered a car accident. The axial MPR image (a) (4-mm thickness) shows an eccentric dilatation of the right common carotid artery (arrow) with the typical "snowman" appearance; the pseudoaneurysm (arrow) is better depicted on the coronal MIP reconstruction (b) (10 mm thickness). A grade IV lesion of the left common carotid artery was also present. No clinical-radiological risk factors for BCVI were present. The patient underwent anti-aggregation and no neurological deficit occurred. c, d A

regarding the evaluation of carotid, vertebral and basilar arteries (graded from 0 to 5). The strength of agreement was evaluated as follows: $<0.20$ poor, $0.21-0.40$ fair, $0.41-$ 0.60 moderate, $0.61-0.80$ good, $0.81-1.0$ excellent.

Categorical variables were expressed as absolute frequencies and percentages, continuous variables as medians and ranges (minimum and maximum values).

BCVI prevalence was analysed with respect to patient gender and age, lesion type, lesion grade, risk factor presence and risk factor type. Statistical analysis was carried out using non-parametric tests. Statistical significance was stated using the $\chi^{2}$-test, with Yates' correction when appropriate. A $p$ value of less than 0.05 was considered to indicate a significant difference.

Correlation between the number of risk factors present in a patient and the occurrence of BVCI was assessed with the Spearman test with modified standard error ([35]), in order 19-year-old man who had suffered a car accident. The sagittal MPR image (c) shows a tortuous course (arrowheads) of the right internal carotid artery; the presence of two pseudoaneurysms (arrowheads) is better depicted on the curved MPR image (d) (3-mm thickness). Skull base fractures and a grade IV lesion of the left common carotid artery were present. The patient underwent anti-aggregation but neurological deficit related to a left anterior circle ischaemia was already present at the time of MDCT

to take into account the large differences in sample size between patient groups having 1,2,3 or 4 concomitant clinical-radiological risk factors.

\section{Results}

We found an excellent inter-observer agreement in the classification of carotid, vertebral and basilar arteries according to the Biffl grading system $(\mathrm{K}=0.97)$.

Thirty-two out of the 976 patients included in our study $(3.3 \%)$ showed the presence of BCVI at the MDCT examination: 22 out of these 32 patients $(68.8 \%)$ presented the involvement of a single vessel, 10/32 (32.2\%) presented the concomitant involvement of two vessels, whereas none of the included patients presented the involvement of three or more vessels. Therefore, a total of 42 cerebrovascular 

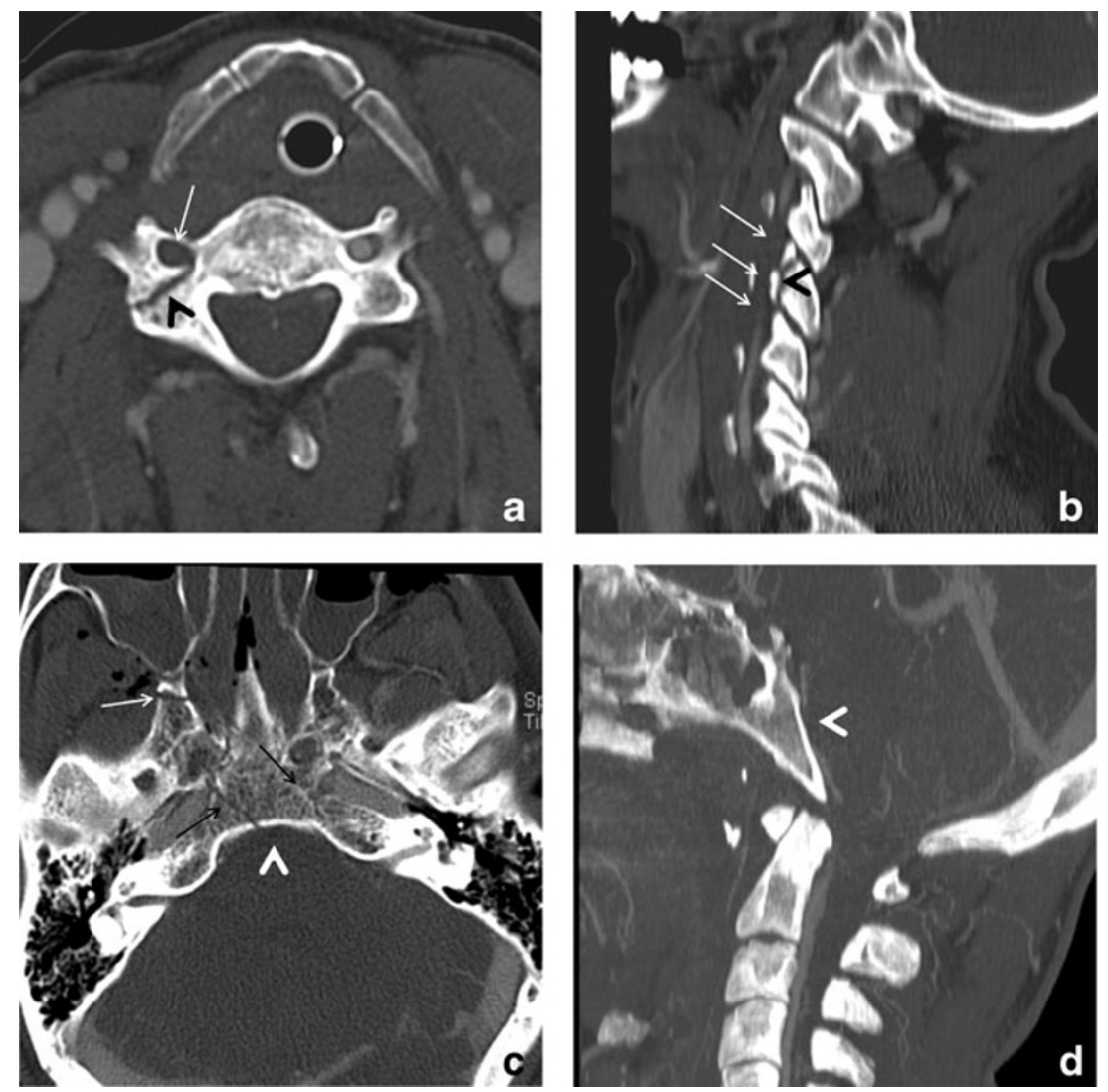

Fig. 3 Blunt cerebrovascular occlusion (grade IV lesions according to the Biffl classification). a, b A 51-year-old man who had suffered a motorcycle accident. The axial MPR image (a) (3-mm thickness) shows the absence of contrast material within the right vertebral artery (arrow) and the coexistence of a C4 lateral mass fracture (arrowhead) involving the right vertebral channel; the same findings (arrows and arrowhead) are clearly detectable on the sagittal MPR image on which the presence of contrast material is detectable within the right vertebral artery upstream and downstream the occlusion. The patient underwent

lesions were observed in our patient population, with an overall prevalence of injured vessels of $4.3 \%$. BCVI involved the carotidal axis (common, internal and external carotid arteries) in 26/42 cases (61.9\%) and the vertebrobasilar axis (basilar and vertebral arteries) in 16/42 cases (38.1\%). Blunt cerebrovascular injuries were classified as grade 1 (Fig. 1a) in 10/42 (23.8 \%) cases, as grade 2 (Fig. 1b-d) in 8/42 (19.0\%) cases, as grade 3 (Fig. 2a-d) in $6 / 42(14.3 \%$ ) cases, as grade 4 (Fig. 3a-d) in $16 / 42$ $(38.1 \%)$ cases and as grade 5 (Fig. $4 a-c)$ in $2 / 42(4.8 \%)$ cases. Data about frequencies of the involved vessels and corresponding lesion grading are reported in Table 1.

Out of the 976 included patients, 247 (25.3\%) showed the presence of one or more clinical-radiological risk factors for BCVI, according to the Memphis criteria [20]. In particular, 134/976 (13.7\%) patients showed the presence of cervical spine fractures, 93/976 (9.5\%) patients presented a Le Fort II or Le Fort III facial fracture and 25/976 (2.6\%)

anti-aggregation and no neurological deficit occurred. c, d A 28-yearold man who had suffered a bicycle accident. The axial MPR image (c) (3-mm thickness) shows multiple skull basis fractures (arrows) involving the clivus too and no contrast material within the basilar artery (arrowhead). Mid-face fractures were also present. The sagittal MIP reconstruction (d) (4-mm thickness) confirms a focal absence of contrast material within the lumen of the basilar artery. The patient did not undergo anti-aggregation because of high haemorrhagic risk and developed a brainstem infarction responsible of a locked in syndrome

of them presented skull base fractures involving the foramen lacerum. Moreover, diffuse axonal injury was suspected on the basis of the brain CT findings in 47/976 (4.8\%) patients. Among the 247 patients presenting risk factors for BCVI, $195(78.9 \%)$ presented a single risk factor, whereas 52 of them $(21.1 \%)$ presented the coexistence of two or more risk factors.

Within the group of 247 patients presenting clinicalradiological risk factors for BCVI, 20/247 (8.1\%) patients showed the presence of BCVI at MDCT (a total of 24 injured vessels has been found within this group of 20 patients, since four of them showed the involvement of two vessels each), whereas 227/247 (92.9\%) patients did not definitely show the presence of BCVI at MDCT.

Among the 729 patients without clinical-radiological risk factors for BCVI, 12/729 (1.6\%) patients showed the presence of BCVI at MDCT (a total of 18 injured vessels has been found within this group of 12 patients, since six of 
Fig. 4 Blunt cerebrovascular transection (grade V lesion according to the Biffl classification). A 24-year-old man who had suffered a motorcycle accident. In the axial MPR image (a) (3-mm thickness) contrast material is clearly recognisable within the left vertebral artery (arrow); moreover, active arterial bleeding causing contrast material extravasation is appreciable next to the left vertebral artery (arrowheads). The same findings are recognisable on the sagittal MPR image (b) (3-mm thickness) where an anterior sliding of $\mathrm{C} 1$ (arrowhead) over $\mathrm{C} 2$ is also appreciable. The coronal MPR image (c) (3-mm thickness) demonstrates that the aforementioned alterations are due to an odontoid process base fracture (type III fracture according to the Anderson classification) (arrowhead). The patient died shortly after the CT
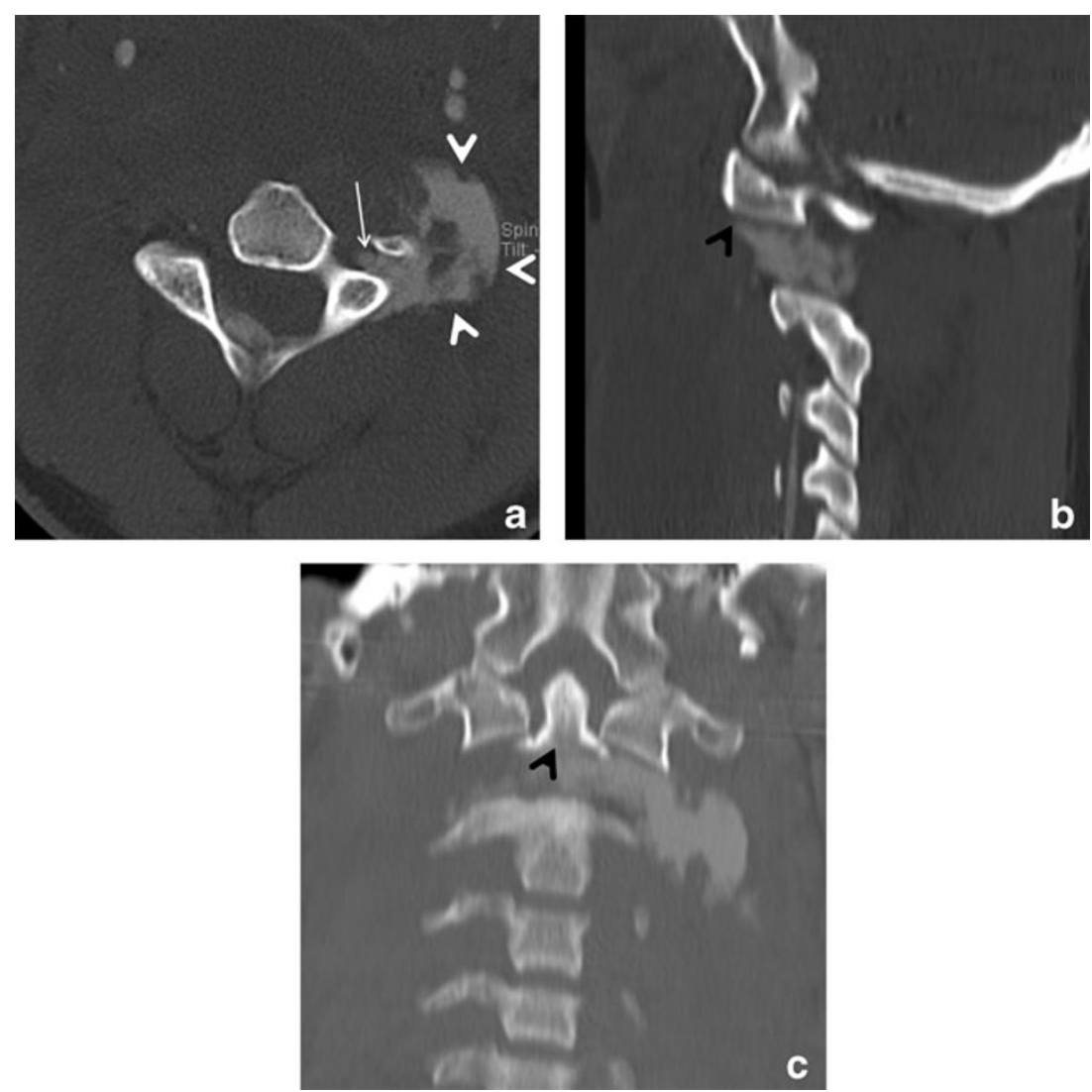

them presented the contemporary involvement of two vessels each), whereas 717/729 (98.4 \%) did not show the presence of BCVI at MDCT.

The comparison of prevalence of patients affected by BCVI in the group presenting risk factors versus the prevalence of patients affected by BCVI in the group without risk factors demonstrated a significant correlation between blunt cerebrovascular injuries and the presence of clinical-radiological risk factors according to the Memphis criteria ( $p=0.009, \chi^{2}$-test with Yates' correction); the strength of correlation was $p<0.0001$ for injuries of the vertebro-basilar axis and $p=0.025$ for carotid arteries injuries (both $\chi^{2}$-test with Yates' correction). Moreover, a statistically significant positive correlation between the number of clinical-radiological risk factors present in each patient and the prevalence of BCVI has been found $(p<0.0002$, Spearman test with modified standard error [35]): the higher is the number of risk factors, the higher is the probability of having a cerebrovascular injury $(p<0.001)$ (Fig. 6).

\section{Discussion}

Our study evaluated over a 7-year period 976 consecutive multi-trauma patients that underwent a whole-body MDCT scan according to our multi-trauma protocol within $12 \mathrm{~h}$ from admission to our emergency department.

Our study showed an extremely high inter-observer agreement $(\mathrm{K}=0.97)$ in the classification of carotid, vertebral and basilar arteries according to the Biffl grading system; this could be a consequence of the high percentage of negative imaging studies $(96.3 \%)$ in our patient population.

BCVIs were present in $3.3 \%$ of our multi-trauma patients. The prevalence of BCVI in our patient population was higher compared with the values reported in the literature, which range from $0.5 \%$ to $2.7 \%$ according to the different patient populations. This discrepancy can be considered a consequence of the particular characteristics of our patient population: indeed, differently from other studies, in our study we included only severely injured patients, classified as multi-trauma at admission to our Emergency Department, instead of all blunt-injured patients, and it is well known that the probability of suffering from BCVI increases with an increase of the Injury Severity Score [1-7].

A total of 42 injured vessels has been found in 32 patients, since ten of them presented the concomitant involvement of two vessels each; in nine out of these ten cases the patient showed symmetrical lesions, regarding the same vessel bilaterally, i.e. either bilateral involvement of the carotidal axis or bilateral involvement of the vertebro-basilar axis, whereas only in one out of ten cases carotid and vertebral arteries were 
Fig. 5 Possible devastating outcomes of untreated BCVIs. A 28-year-old man who had suffered a car accident. The sagittal MIP reconstruction (a) (3-mm thickness) shows a progressive tapering of the left internal carotid artery (arrowheads) that becomes occluded before the intracranial portion. Latero-lateral digital subtraction angiography image (b) shows exactly the same finding as MDCT (arrowheads) with no contrast material within the intracranial portion of left internal carotid artery. The patient showed no risk factors for BCVI and was neurologically asymptomatic at the time of MDCT. Antiaggregation was not undertaken because of the presence of severe abdominal injuries and high bleeding risk. The axial DWI MR image (c) $(b=1,000)$ acquired 2 days later, after the onset of right hemiparesis and aphasia, shows a marked signal hyperintensity in the territory of the left mean cerebral artery representing acute infarction

involved at the same time. This fact might be related to the different trauma dynamics involving carotid and vertebrobasilar arteries; indeed, carotid arteries are usually injured as a result of a stretching mechanism due to hyperextension and rotation of the neck, whereas vertebro-basilar arteries, which are strictly surrounded by bony structures, are more commonly directly damaged by bone fractures.

A review of the original MDCT radiological reports of the patients affected by BCVI showed that lesions had been originally missed in 3/32 (9.4\%) patients. The missed injuries where one grade 1 lesion of right vertebral artery, one grade 1 lesion of left internal carotid artery and one grade 2 lesion of left vertebral artery. This result highlights the possible difficulties in diagnosing BCVI, particularly in low grade cases.

Our study confirmed the existence of a significant correlation between the presence of clinical-radiological risk factors as proposed by the Memphis group and the eventuality of suffering from blunt cerebrovascular injury $(p=0.009)$. To be more precise, we found a stronger correlation between the presence of risk factors and vertebro-basilar injuries than between the presence of risk factors and carotid arteries injuries ( $p<0.001 \mathrm{vs}$ $p=0.025$ ); this result might also be related with the abovementioned different trauma dynamics involving carotid and vertebro-basilar arteries, the latter usually being directly injured by bone lesions and, therefore, strongly associated to cervical spine fractures. The risk factors proposed in the Memphis approach, however, do not only represent possible direct vascular injury pathways, like in case of cervical and skull base fractures, but they also may represent an index of the severity of the cranio-cervical trauma, like in case of Le Fort II and III fractures and diffuse axonal injury; this can explain why carotid arteries injuries are also significantly associated with the abovementioned risk factors, although not as strongly as vertebral ones. Moreover, our study showed the existence of a positive correlation between the number of clinical-radiological risk factors and the probability for a patient of having a blunt
Table 1 Blunt cerebrovascular injuries (BCVIs) (42 lesions): number of involved vessels correlated to the grading of the lesions

\begin{tabular}{lllllll}
\hline & Grade 1 & Grade 2 & Grade 3 & Grade 4 & Grade 5 & Total \\
\hline Common and internal carotid arteries & 6 & 7 & 6 & 7 & 0 & 26 \\
Vertebral and basilar arteries & 4 & 1 & 0 & 9 & 2 & 16 \\
Total & 10 & 8 & 6 & 16 & 2 & 42 \\
\hline
\end{tabular}




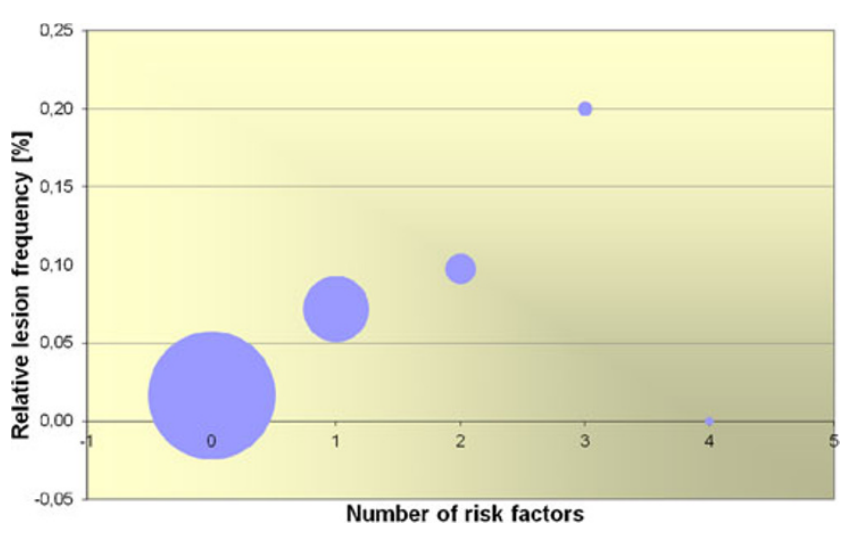

Fig. 6 Relative frequency of BCVI correlated with the number of risk factors. The $x$-axis shows the number of risk factors characterising each patient group, whereas the $y$-axis reports the probability (in percentage) of having a BCVI for each group. The area of the blue circles is correlated with the size of each patient group (729 patients with no risk factors, 195 patients with one risk factor, 41 with two risk factors, 10 with three risk factors, 1 with four risk factors)

cerebrovascular injury: the higher is the number of risk factors that are contemporary present in the same time, the higher is the probability for that patient of having a cerebrovascular injury $(p<0.0002)$ (Fig. 6).

Nevertheless, according to our data $37.5 \%$ of the patients affected by BCVI did not show any of the above-mentioned clinical-radiological risk factors. As a result, a screening examination for the presence of blunt cerebrovascular injuries cannot be exclusively reserved for multi-trauma patients presenting clinical-radiological risk factors for BCVI, because such an approach would miss an early diagnosis in more than one-third of positive cases, denying therefore the possibility of the instauration of a prompt therapy and increasing the probability of catastrophic outcomes. Therefore, according to our experience, all multi-trauma patients should be screened for the presence of BCVI, despite the absence of risk factors. With BCVI being relatively uncommon, showing an incidence of $3.3 \%$, the selected screening method should be cost effective and not time consuming or invasive; in order to achieve this goal, a post-contrast acquisition of the neck can be easily integrated into the wholebody MDCT protocol performed in haemodynamically stable multi-trauma patients at admission without additional contrast material administration nor an additional dose exposure, because it substitutes the plain acquisition of the cervical spine.

The main limitation of our study is due to its retrospective design. Another weakness is the absence of a comparison of MDCT with the gold standard, which for BCVI is still considered to be DSA. Anyway, the recent literature $[30,32]$ demonstrated that MDCT can be considered an acceptable initial means of screening for BCVI, without statistically significant differences in accuracy compared with DSA. In our opinion, an invasive, costly and time consuming diagnostic investigation like DSA could be reserved to patients with high clinical suspicion of BCVI and negative MDCT findings.

In conclusion, we strongly suggest to screen all multitrauma patients for the presence of BCVI by including a bolus-timed arterial scan of the neck into the whole-body MDCT protocol performed on haemodynamically stable patients at admission in order to early detect and early treat BCVIs.

Acknowledgments We would like to thank Professor Agostino Tarsitano for his help in the statistical analysis.

Open Access This article is distributed under the terms of the Creative Commons Attribution License which permits any use, distribution, and reproduction in any medium, provided the original author(s) and the source are credited.

\section{References}

1. Biffl WL, Moore EE, Elliott JP et al (2000) The devastating potential of blunt vertebral arterial injuries. Ann Surg 231 (5):672-681

2. Mutze S, Rademacher G, Matthes G, Hosten N, Stengel D (2005) Blunt cerebrovascular injury in patients with blunt multiple trauma: diagnostic accuracy of duplex Doppler US and early CT angiography. Radiology 237(3):884-892

3. Miller PR, Fabian TC, Bee TK et al (2001) Blunt cerebrovascular injuries: diagnosis and treatment. J Trauma 51(2):279-285, discussion 85-6

4. Schneidereit NP, Simons R, Nicolaou S et al (2006) Utility of screening for blunt vascular neck injuries with computed tomographic angiography. J Trauma 60(1):209-215, discussion 15-6

5. Cothren CC, Moore EE, Ray CE Jr et al (2005) Screening for blunt cerebrovascular injuries is cost-effective. Am J Surg 190(6):845-849

6. Sliker CW (2008) Blunt cerebrovascular injuries: imaging with multidetector CT angiography. Radiographics 28(6):1689-1708, discussion 709-10

7. Stein DM, Boswell S, Sliker CW, Lui FY, Scalea TM (2009) Blunt cerebrovascular injuries: does treatment always matter? J Trauma 66(1):132-143, discussion 43-4

8. Fusco MR, Harrigan MR (2011) Cerebrovascular dissections-a review part I: Spontaneous dissections. Neurosurgery 68(1):242257, discussion 57

9. Fusco MR, Harrigan MR (2011) Cerebrovascular dissections: a review. Part II: blunt cerebrovascular injury. Neurosurgery 68 (2):517-530, discussion 30

10. Cogbill TH, Moore EE, Meissner M et al (1994) The spectrum of blunt injury to the carotid artery: a multicenter perspective. J Trauma 37(3):473-479

11. Rodriguez M, Tyberghien A, Matge G (2001) Asymptomatic vertebral artery injury after acute cervical spine trauma. Acta Neurochir (Wien) 143(9):939-945

12. Fabian TC, Patton JH Jr, Croce MA, Minard G, Kudsk KA, Pritchard FE (1996) Blunt carotid injury. Importance of early diagnosis and anticoagulant therapy. Ann Surg 223(5):513-522, discussion $22-5$

13. Franz RW, Willette PA, Wood MJ, Wright ML, Hartman JF (2012) A systematic review and meta-analysis of diagnostic screening criteria for blunt cerebrovascular injuries. J Am Coll Surg 214(3):313-327 
14. Burlew CC, Biffl WL, Moore EE, Barnett CC, Johnson JL, Bensard DD (2012) Blunt cerebrovascular injuries: redefining screening criteria in the era of noninvasive diagnosis. J Trauma Acute Care Surg 72(2):330-335, discussion 6-7, quiz 539

15. Mokri B (1990) Traumatic and spontaneous extracranial internal carotid artery dissections. J Neurol 237(6):356-361

16. Krajewski LP, Hertzer NR (1980) Blunt carotid artery trauma: report of two cases and review of the literature. Ann Surg 191 (3):341-346

17. Mayberry JC, Brown CV, Mullins RJ, Velmahos GC (2004) Blunt carotid artery injury: the futility of aggressive screening and diagnosis. Arch Surg 139(6):609-612, discussion $12-3$

18. DiCocco JM, Fabian TC, Emmett KP et al (2011) Optimal outcomes for patients with blunt cerebrovascular injury (BCVI): tailoring treatment to the lesion. J Am Coll Surg 212(4):549-557, discussion 57-9

19. Cothren CC, Moore EE, Biffl WL et al (2004) Anticoagulation is the gold standard therapy for blunt carotid injuries to reduce stroke rate. Arch Surg 139(5):540-545, discussion 5-6

20. Miller PR, Fabian TC, Croce MA et al (2002) Prospective screening for blunt cerebrovascular injuries: analysis of diagnostic modalities and outcomes. Ann Surg 236(3):386-393, discussion 93-5

21. Harrigan MR, Weinberg JA, Peaks YS et al (2011) Management of blunt extracranial traumatic cerebrovascular injury: a multidisciplinary survey of current practice. World J Emerg Surg 6:11

22. Biffl WL, Ray CE Jr, Moore EE et al (2002) Treatment-related outcomes from blunt cerebrovascular injuries: importance of routine follow-up arteriography. Ann Surg 235(5):699-706, discussion $706-7$

23. Parodi JC, Schonholz C, Ferreira LM, Bergan J (1999) Endovascular stent-graft treatment of traumatic arterial lesions. Ann Vasc Surg 13(2):121-129

24. Price RF, Sellar R, Leung C, O’Sullivan MJ (1998) Traumatic vertebral arterial dissection and vertebrobasilar arterial thrombosis successfully treated with endovascular thrombolysis and stenting. AJNR Am J Neuroradiol 19(9):1677-1680
25. Attigah N, Kulkens S, Zausig N et al (2009) Surgical therapy of extracranial carotid artery aneurysms: long-term results over a 24year period. Eur J Vasc Endovasc Surg 37(2):127-133

26. Biffl WL, Ray CE Jr, Moore EE, Mestek M, Johnson JL, Burch JM (2002) Noninvasive diagnosis of blunt cerebrovascular injuries: a preliminary report. J Trauma 53(5):850-856

27. Bromberg WJ, Collier BC, Diebel LN et al (2010) Blunt cerebrovascular injury practice management guidelines: the Eastern Association for the Surgery of Trauma. J Trauma 68(2):471-477

28. Biffl WL, Cothren CC, Moore EE et al (2009) Western Trauma Association critical decisions in trauma: screening for and treatment of blunt cerebrovascular injuries. J Trauma 67(6):1150-1153

29. Langner S, Fleck S, Kirsch M, Petrik M, Hosten N (2008) Wholebody CT trauma imaging with adapted and optimized CT angiography of the craniocervical vessels: do we need an extra screening examination? AJNR Am J Neuroradiol 29(10):1902-1907

30. Sliker CW, Shanmuganathan K, Mirvis SE (2008) Diagnosis of blunt cerebrovascular injuries with 16-MDCT: accuracy of wholebody MDCT compared with neck MDCT angiography. AJR Am J Roentgenol 190(3):790-799

31. Fleck SK, Langner S, Baldauf J, Kirsch M, Kohlmann T, Schroeder HW (2011) Incidence of blunt craniocervical artery injuries: use of whole-body computed tomography trauma imaging with adapted computed tomography angiography. Neurosurgery 69(3):615-623, discussion 23-4

32. Rademacher G (2008) Gefaessdissektion der hirnversorgenden Arterien: Screening im Rahmen der Ganzkoerpercomputertomographie. Trauma und Berfufskrankheit 3(10):182-186

33. Biffl WL, Moore EE, Offner PJ et al (1999) Optimizing screening for blunt cerebrovascular injuries. Am J Surg 178(6):517-522

34. Hughes KM, Collier B, Greene KA, Kurek S (2000) Traumatic carotid artery dissection: a significant incidental finding. Am Surg 66(11):1023-1027

35. Cook A, Osler T, Gaudet M, Berne J, Norwood S (2011) Blunt cerebrovascular injury is poorly predicted by modeling with other injuries: analysis of NTDB data. J Trauma 71(1):114-119

36. Biffl WL, Moore EE, Offner PJ, Brega KE, Franciose RJ, Burch JM (1999) Blunt carotid arterial injuries: implications of a new grading scale. J Trauma 47(5):845-853 